
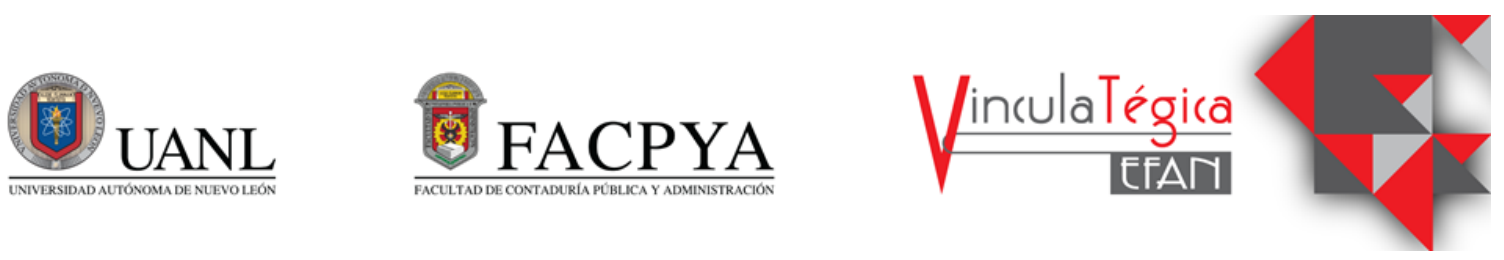

\title{
El modelo triple hélice en el contexto de la Industria 4.0
}

\author{
Cristina Isabel Laines Alamina ${ }^{1}$, Ivonne Jannette Silva Almanza ${ }^{2}$ y Luz Tania Guajardo \\ Muñoz ${ }^{3}$ \\ ${ }^{1}$ Universidad Autónoma de Nuevo León, cristina.laineslm@uanl.edu.mx, Cd. Universitaria, San Nicolás de \\ los Garza, N.L. 8112-76150 \\ ${ }^{2}$ Universidad Autónoma de Nuevo León, Ivonne.silvalm@uanl.edu.mx, Cd. Universitaria, San Nicolás de los \\ Garza, N.L. 8113-009058 \\ ${ }^{3}$ Universidad Autónoma de Nuevo León, luz.guarjardomnz@uanl.edu.mx, Cd. Universitaria, San Nicolás de \\ los Garza, N.L. 8182-872222
}

Información del artículo revisado por pares

Fecha de aceptación: junio-2021

Fecha de publicación en línea: diciembre-2021

DOI: https://doi.org/10.29105/vtga7.1-93

\section{Resumen}

En este artículo abordamos la basé teórica del modelo de la Triple Hélice que involucra la vinculación existente de cooperación entre las Universidades - Empresas y Gobierno. Cuando las empresas, gobiernos y universidades trabajan de manera conjunta, se puede desarrollar una fuerza laboral precisa y necesaria para impulsar el crecimiento económico y garantizar que la fuerza laboral en su conjunto esté preparada para prosperar en la próxima cuarta revolución industrial. El análisis realizado de esta teoría deja claro que existe un amplio espectro que contempla la Triple Hélice como un modelo vigente en Asia, America Latina y Europa, necesario para mejorar la participación e involucramiento en estos ámbitos.

Palabras clave: Triple Hélice, Industria 4.0, Habilidades, Gestión, Innovación.

\section{Abstract}

In this article we address the theoretical basis of the Triple Helix model that involves the existing link of cooperation between the Universities - Companies and the Government. When businesses, governments and universities work together, a precise and necessary workforce can be developed to fuel economic growth and ensure that the workforce as a whole is prepared to thrive in the next fourth industrial revolution. The analysis carried out on this theory makes it clear that there is a wide spectrum that contemplates the Triple Helix as a current model in Asia, Latin America and Europe, necessary to improve participation and involvement in these areas.

Keywords: Triple Helix, Industry 4.0, Skills, Management, Innovation.

JEL: M15 IT Management, 03 Innovation, 032 Managements of technological innovation and $\mathrm{R}+\mathrm{D}, \mathrm{O} 41$ One, two and multisector, growth models. 


\section{INTRODUCCIÓN}

La aparición de nuevas formas de cooperación y de hacer negocio, después del escenario suscitado por la pandemia del COVID-19 ha originado que las empresas, enfoquen sus esfuerzos en desarrollar tecnologías que les permitan entrar en niveles de competitividad oportunos.

El Gobierno, en cooperación con las Universidades y los clústeres empresariales, han manifestado que la vinculación de estos tres grandes entes, toman suma importancia para el fortalecimiento del conocimiento, relaciones y por consecuencia la generación de ambientes que den parte al desarrollo que se necesita a partir de la generación de nuevos conocimientos.

En el contexto actual las investigaciones existentes son planteadas a partir de una óptica de necesidad de cooperación entre las organizaciones, con la finalidad de estar en posibilidades de desarrollar nuevas estrategias y productos, que coadyuven para generar y fortalecer, nuevas y mejores tecnologías, así mismo generar diversificación y adquisición de nuevos conocimientos para el desarrollo de innovación e investigación en las organizaciones.

Los orígenes del modelo Triple Hélice nacen en la perspectiva de la universidad empresarial que emerge en los Estados Unidos, donde hay un fuerte arraigo de colaboraciones entre elementos de los entornos, académicos, industriales y universitarios (Etskowitz,1989). Dichas relaciones en estos ámbitos permiten explicar modelos para demostrar la importancia de la innovación como factor esencial en este nuevo tipo de economías, que es posible dada las TIC's y procesos de globalización en los mercados. Lo anterior debido a que la innovación de los sistemas basados en conocimientos puede ser considerados como enfoques teóricos de un dinamismo completo desde diferentes ángulos y con metas potencialmente diferentes. (Leyersdorff, 2005)

En ese tenor de innovación, adaptabilidad, y cambios, la industria 4.0 es parte primordial de esta transformación y se integran para hacer surgir sistemas innovadores de gestión empresarial y maneras de realizar negocios, que faciliten la optimización de los procesos para alcanzar mayor eficiencia y generar valor.

La trascendencia de esta revolución tecnológica tiene tal magnitud, que está incidiendo en las diversas esferas de las organizaciones, desde la producción hasta la investigación y desarrollo, la gestión y el soporte al cliente. Está modificando la visión y actuación empresarial. La Industria 4.0 es ya considerada como un proceso sistémico de innovación que rediseña y da nuevos rumbos a los modelos de gestión empresarial y brinda nuevos horizontes y perspectivas, integrados al entorno de la organización. Cortés, C. B. Y., Landeta, J. M. I., \& Chacón, J. G. B. (2017).

\section{MARCO TEÓRICO}

La innovación se distingue por ser novedosa y usualmente impredecible, aunque ello no signifique que no se pueda hacer esfuerzo para controlar y organizar la generación de sistemas de innovación, de ahí que el interés no se centre en el concepto aislado de la innovación sino en la innovación de la innovación (Cárdenas, y Ríos 2016).

Diversos autores aseguran que, al exterior de cadena de valor, los eslabones entre las empresas con las universidades son de menor frecuencia e intensidad, tal como señala Grotz y Braun (1997), en cambio, la faceta reactiva de las innovaciones en las pequeñas y medianas empresas entorpece a aún más la suma e incorporación de agentes de innovación y desarrollo que tienen una actitud investigadora dinámica y propositiva orientada a largo plazo (Lavía et al., 2011).

El modelo de la Triple Hélice, desarrollado por Etzkowitx y Leydesdorff (1995, 2000) plantea diversos medios de interconexión para sus integrantes, representados las empresas, las universidades y el gobierno.

De esa vinculación nace el interés de analizar y contextualizar la importancia y 
relación de esos nuevos escenarios post pandemia y como su transformación permite desarrollar y enfocar la industria hacia nuevas formas de hacer negocios.

Tal como podemos observar en la Figura 1, la visualización del modelo Triple Hélice.

\section{Figura.1}

Elaboración propia. Fuente Etzkowitz y

Leydesdorff (2000)
A través de este modelo se visualiza a la Universidad como generador de conocimiento y marca un rol fundamental de vinculación entre los entes representado por el gobierno y la industria y la forma en que estos se conjugan para hacer surgir formas innovadoras en las organizaciones como fuente de origen de conocimiento y su desarrollo.

Así mismo se considera a este modelo TH como

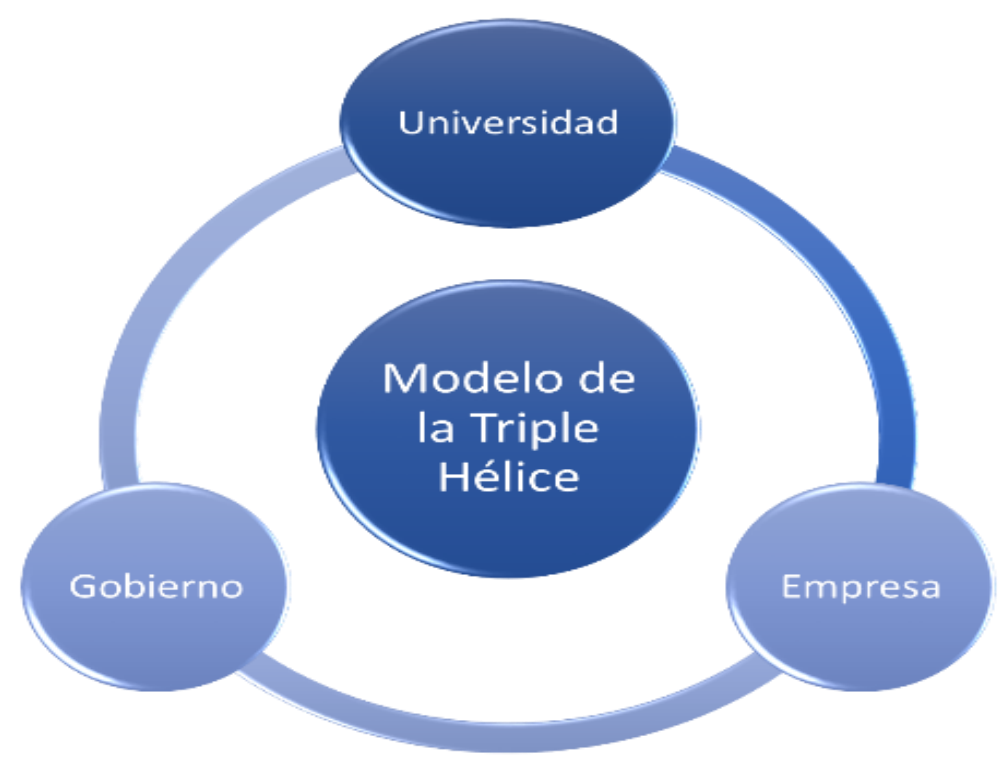

La industria 4.0 también conocida como manufactura avanzada, es el resultado de la evolución de los sistemas formalmente incorporados a los sistemas ciber/físicos. Así mismo significa el arribo de la Cuarta Revolución industrial, que denota el camino hacia el internet de las cosas, datos y servicios.

La inteligencia descentralizada ayuda a instituir redes de objetos inteligentes y facilita la gestión de procesos independientes, con la interrelación entre el mundo virtual y el mundo real (Baheti y Gill, 2011), brindando de esta manera un nuevo escenario en los productos y diversos procesos de producción.

\subsection{El modelo de la Triple Hélice}

Este modelo tiene sus orígenes en estudios realizados por Etzkowitz y Leydesdorff (1997). un proceso intelectual orientado a vislumbrar que los lazos que se den entre las vinculaciones de la universidad, y la sociedad, evolucionen, sin embargo, también se distinguen por la injerencia de la universidad en los procesos económicos y sociales. Chang Castillo, H.G. (2010).

La Triple Hélice toma como punto de partida que la "innovación no es una unidad estable de análisis sino una unidad de operación en una interfaz" (Leydesforff, 2001:2). Los sistemas de innovación se dan derivados de los resultados de la relación de los intercisos de los tres subtemas involucrados. Lo cual facilita la superación del marco de los sistemas de innovación nacional, como una unidad de análisis relevante y que considera a la innovación de los sistemas emergentes en función de un sub red, sobre las redes que los constituyen (disciplinas, industria y gobiernos) (Leydesforff y Etzkowitz, 2001). 
El modelo de la $\mathrm{TH}$, genera que existe un vínculo entre las diversas disciplinas y áreas de conocimiento, que es ahí donde la universidad funge con la tarea estratégica y forma parte fundamentar para generar las bases solidad de relaciones con las empresas. Chang Castillo, H.G(2010).

En el modelo TH los roles de la universidad y del gobierno ya no se consideran estáticos o solo de colaboración si no requieren una interacción entre las diferentes funciones para generar dinamismo e innovación.

Bueno, J. L. M. (2017) señala que los estudios se basan principalmente en el análisis de las interacciones y los efectos en procesos de innovación, en la construcción de indicadores y medición de dinámicas y en nuevas contribuciones teóricas, mientras otros se centran en la configuración como modelo por aplicarse a otros contextos temáticos y como propuesta para diseño de políticas de innovación.

De acuerdo a lo establecido por a Etzkowitz y Klofsten (2005) el modelo de la Triple Hélice consta de tres elementos fundamentales: Implica una mayor influencia del papel de la universidad en los procesos de innovación, a la par de la industria y el gobierno, bajo la premisa de la sociedad del conocimiento. Existe un movimiento entre las relaciones de vinculación entre los ámbitos institucionales en lo que la política de innovación es cada vez más un resultado de la interacción y no una receta de gobierno. Cada aspecto institucional funge un rol en los otros que operan en sus ejes y en su función tradicional. Las universidades que juegan un rol empresarial que toman papeles tradicionales de la industria y el gobierno que es la figura central para la innovación en diversos sectores sociales.

La tendencia actual hace hincapié en la internacionalización de los sistemas de innovación mediante el surgimiento de redes de internacionalización, vislumbrando que el sistema de la producción del conocimiento es cada vez más globalizado (Smith y Leydesdorff, 2015.)

Así mismo Etzkowitz (2002) establece un modelo para la intervención en las políticas públicas para el desarrollo regional basado en un esquema de tres etapas consecutivas:

a) Espacio de conocimiento: se centra principalmente en entornos de innovación regionales, donde actores trabajan principalmente para mejorar las condiciones locales de la innovación mediante la concentración de actividades que estén relacionadas y operaciones relevantes.

b) Espacio de consenso: se generan ideas y estrategias en una $\mathrm{TH}$, de variadas relaciones reciprocas entre sectores institucionales, académicos (públicos y privados)

c) Espacio de innovación: son los intentos de realizar objetivos articulados en la fase anterior, es centrar y establecer o atraer capital, riesgo público y privado para combinar el capital, el conocimiento técnico y el conocimiento empresarial.

Sin embargo, es importante señalar que el modelo se planteó de manera inicial como ya ha sido mencionado como el vínculo de universidad, empresa y gobierno, sin embargo, ha atravesado por tres etapas:

1. La triple hélice I: Es considerada la primera versión de este modelo y señala que, en la administración general de la figura del Gobierno, se centran las relaciones entre la académica y la industria. En esta versión se determinan asociaciones de similitud con el triángulo de Sábato.

2. La triple hélice II. En esta segunda versión se dividen las diversas esferas institucionales, afirmando su autonomía. En esta perspectiva se limita por las fuertes barreras entre una y otra, además de las relaciones previamente existentes.

3. La triple hélice III. En esta se versión establece una infraestructura para la generación de nuevo conocimiento, en la cual se superponen las esferas institucionales, de manera que cada una toma el rol de la otra. En estos espacios de interfaz emergen organizaciones híbridas $o$ interfaces, y un área llamada Red Trilateral y de 
Organizaciones Híbridas. Lo anteriormente descrito de acuerdo a la recopilación realizada por Hernández, L. C., Verástegui, J. L., \& Melo, N. A. P. (2014). Castillo Hernández, en la evolución y antecedentes de su documento.

Según lo señala Chang, H. (2010), "en la mayoría de los países y regiones se está actualmente tratando de lograr alguna forma de Triple Hélice III, El objetivo es lograr un entorno innovador que consiste principalmente en que la Universidad de las empresas spin off, que son iniciativas para el desarrollo económico basado en el conocimiento y las alianzas estratégicas entre las empresas y grupos de investigación académica."

Es importante resaltar la afinidad de la Triple Hélice para sumar esfuerzos en la solución de problemáticas actuales en ámbitos medio ambientales y sociales (Ezkowitz y Zhou, 2006). la intención es trasladar el modelo de la TH hacia la generación de otras triples hélices, que aborden y se ocupen de dar atención a problemáticas sociales, necesarias de atención en los diversos estratos y de igual poner foco de atención a las soluciones de índole científica y tecnológica, como lo ha descrito De la FE T. (2009).

Por un lado, exportando el modelo hacia la creación de otras triples hélices que atiendan a problemas sociales preponderantes en distintas partes del mundo y por otro lado poniendo a trabajar a las entidades $\mathrm{TH}$ en soluciones científicas y tecnológicas, tal como señala De la Fe T. (2009).

Y es precisamente en ese tenor que toma forma e importancia la vinculación de la Industria 4.0 con este modelo de innovación para lograr el desarrollo económico de las regiones, con la firme intención de crear nuevos conocimientos, fortalecer la creación de nuevas empresas e incentivar la innovación y el desarrollo de las TICs.

\section{a. La industria 4.0: definición y alcances.}

La industria 4.0 también conocida como manufactura avanzada, es el resultado de la evolución de los sistemas integrados a sistemas ciber-físicos. Es la llegada de esta industrial, tiende el camino hacia el IoT, en cuanto a datos y servicios refiere. Es esta inteligencia que de manera descentralizada la que apoya en la creación de redes en objetos inteligentes; además de que da pie a que la gestión de procesos de manera independiente haga una interacción entre el mundo virtual y el real (Baheti y Gill, 2011), brindando de esta manera un nuevo escenario en los productos y diversos procesos de producción.

El concepto como tal de industria 4.0 nace en Alemania en el año 2011 ya hace referencia a una a una política económica de gobierno, que se basa principalmente en estrategias de alta tecnología política económica gubernamental, basada en estrategias de alta tecnología.

Como podemos ver a continuación en la Figura 2 , los alcances de la Industria 4.0 y su marco institucional.

\section{Figura 2.}

Elaboración propia. Sáenz, C. C. (2016). Industria 4.0.

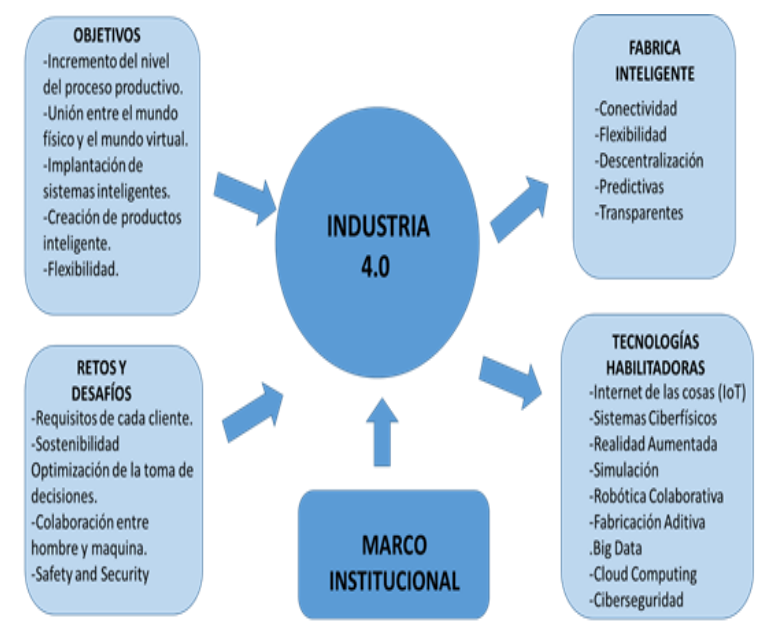

La industria 4.0 se enfoca en tres aspectos esenciales: individualización, descentralización y en la creación de redes. Individualización: También denominada personalización masiva: es una estrategia de producción centrada en 
producir en masa productos, en su mayoría en procesos poco flexibles y la integración de los miembros de la cadena de suministro a través de la cadena de valor (Pine,1993)Descentralización: es un modo de organización de la producción en virtud del cual se hace en encargo a terceros de determinadas partes u operaciones del proceso productivo (Ermida Uriarte y Colotuzzo, 2009)Creación de redes: se refiere a la capacidad de interacción de diversos entes involucrados y con intereses similares, como es el caso de este documento que vincula el modelo Triple Hélice y su vinculación en la Industria 4.0 Cortés, C. B. Y., Landeta, J. M. I., \& Chacón, J. G. B. (2017).

La industria 4.0 involucra el desarrollo de sistemas, el internet de las cosas (IoT), el big data, la inteligencia artificial, digitalización, entre otros que al conjuntarse generan cambios de manera trascendental tanto en la industria, así como en el comportamiento de los consumidores y la forma en que las organizaciones hacen negocios.

\subsubsection{Objetivos, retos y desafíos de la Industria 4.0}

1. Objetivos: de extremo a extremo la digitalización dará como resultado que en las fases productivas se generen instalaciones independientes, así como de cadenas de producción en las que estas puedan ser gestionables de manera autónoma, aumentando así su cadena de valor, esto hacia niveles nuevos de control y organización; esto derivado de la interacción de la investigación, diseño, desarrollo, producción logística y prestación de servicios.

En virtud de ello, lo que refiere a la integración de la industria entre lo virtual y lo físico, significará la conexión entre las personas, los objetos y los sistemas. Lo que dará como resultado los Sistemas Ciber físicos, estos son base para que estén disponibles y circulen en tiempo real dentro de un conjunto de información que se genera en los diferentes procesos. Dando como resultado una retroalimentación y conexión que permite la optimización de la cadena de valor, ya que refleja una organización autónoma, esto para una mejor toma de decisiones lo que la hará soporte de dicha auto organización.

Por tanto, esta implementación de procesos o sistemas inteligentes los asiste. Esto permitirá trabajar con grandes volúmenes de datos, ya que proporcionará valor agregado, lo que dará como resultado que tenga capacidad de: 1) optimizar redes y un mantenimiento preventivo; 2) restaurar el sistema, esto deriva en la capacidad de recuperar de manera rápida y eficiente el sistema cuando se presenten desastres naturales que impidan su correcto funcionamiento;3) aprendizaje, conocimiento que proviene de la experiencia de las funcionalidades anteriores que dan como resultado al aumento de la velocidad de respuesta, ya que lo realizan de manera independiente. Evans, $\mathrm{P}$ y Annunziata, M. (2012).

De esta manera se garantiza la flexibilidad que será posible tener respuesta más rápida, respondiendo de manera eficiente a las necesidades de los clientes, desde la fase de inicio hasta su puesta en marcha.

2. Retos y desafíos: Es necesario reinventarse en esta industria, que se basa completamente en digitalizar el proceso productivo; esto para enfrentar los retos que se avecinan.

En un futuro la industria llevará a clientes a colaborar de manera activa en etapas de desarrollo de los productos, esto para que puedan integrarse de manera específica criterios en cada una de ellas como la planificación, diseño, configuración, fabricación y el funcionamiento, incluyendo modificaciones de última hora. Desde este nuevo enfoque, se le asigna a un cliente el papel del consumidor, pero también como diseñador. Con ello las organizaciones podrán tener acceso a la información que ellos mismos generan, dándoles la oportunidad de que lo 
conozcan mejor y con ello hacer más fácil la oferta de un producto totalmente diferente y único. Esto con el fin de que sean más funcionales individualmente. (Spri, 2015a).

En lo que, a la sostenibilidad y eficiencia en los recursos se refiere, esto ya es algo obligado por la industria y no de manera opcional.

Es en Europa donde existe conciencia de que en un futuro dependerá de la industria y la economía el mantener accesos a fuentes de energía- que son de vital interés- de ahí que se centren principalmente en disminuir consumos y encontrar alternativas (Comisión Europea, 2013).

Otro reto importante dentro de la industria es optimizar el proceso de toma de decisiones. En estos últimos tiempos, unos de los más grandes retos de esta industria es recopilar la mayor cantidad de datos posibles; aunque estas grandes cantidades de datos difícilmente llega a ser utilizado en su mayoría; por lo que el objetivo hoy día es dotar a las máquinas, fábricas y dispositivos de la inteligencia ya que estas tendrán una mayor capacidad en la recolección de grandes cantidades de datos de manera que estos sean útiles para tomar decisiones. En resumen, en esta era de la digitalización el reto será desarrollar sistemas de desarrollo de software y herramientas de análisis que puedan transformar estos datos en información útil y valiosa.

El cuarto reto de vital relevancia es la interacción hombre-máquina. En esta industria que revolucionara el futuro se pretende que esta interacción rediseñe las actividades de cada uno. De manera que los robots serán asignados para tareas de rutinas pesadas y repetitivas, como estas que obliguen a una mayor exactitud en la elaboración de un producto y esto permitirá redefinir el rol de los trabajadores, permitiéndoles cualificarlos en tareas de diseño, creación, programación y toma de decisiones inclusive controlando sus robots. Kagermann, H.; Wahlster, W. y Helbig, J. (2013).

3. Fabrica Inteligente: del Val Román, J. L. (2016) comenta que este cuarto pilar está formado por unidades de producción inteligentes y están ligados a un ecosistema; del cual están conscientes de su estado actual y limitaciones. Cada una de las etapas o módulos pueden llegar a obtener la información que necesiten; esta fábrica es capaz de convertirse en una red que pueda generar agentes que toman decisiones óptimas localmente.

\section{En cuanto a las Tecnologías}

Habilitadoras; también menciona que en estas se poya la Industria 4.0; además de ser la base para los ingenieros en informática; esto hace posible que puedan obtener competencias para enfrentar y encabezar a esta nueva revolución. Las tecnologías móviles; son pilar de a IoT; todo ello en tiempo real; computo en la nube tiene infraestructura y aplicaciones que se ofrecen mediante redes privadas o redes públicas. La nube permite el flujo de datos se dé sin fronteras y evita invertir en infraestructura que aumenta su capacidad además de que la haga flexible y sin precedentes.

Las implicaciones y alcances de esta tecnología definitivamente tendrán un manejo complicado respecto a la cuantificación; pero no así en la optimización en cuanto a sistemas de fabricación, logrando una reducción en los tiempos de desarrollos nuevos y menores costos en cuanto a los procesos. Permitiendo llegar a una optimización y automatización de las máquinas, permitiendo así su capacidad de mantenerse y adaptarse de manera automática.

El poder tener acceso a la información en tiempo real facilita que se puedan crear redes de cooperación, para un proceso de mejor toma de decisiones; permitiendo el aprendizaje y una mejora continua. Cortés, C. 
B. Y., Landeta, J. M. I (2017)

Rubiano (2020), señala que no se sabe con certeza como irá evolucionando la Industria 4.0; ya que a la velocidad en que viaja la invención hace complicado el poder prevenir el alcance que tendrán estas a largo plazo. Definitivamente es claro que el resultado debe ser integral, de manera que se involucre a los de manera flexible sin necesidad de un esfuerzo administrativo. Según datos de la OCDE en el 2014 las empresas mexicanas utilizaban la nube solo en un 9\% (Stezano \& Casalet. 2020).

\section{Tabla 1}

Recursos Aprobados para la función Ciencia,

\begin{tabular}{|c|c|c|c|c|c|c|}
\hline $\mathbf{2 0 1 5}$ & $\mathbf{2 0 1 6}$ & $\mathbf{2 0 1 7}$ & $\mathbf{2 0 1 8}$ & $\mathbf{2 0 1 9}$ & $\mathbf{2 0 2 0}$ & $\mathbf{2 0 2 1}$ \\
\hline 81.3 & 75.8 & 61.5 & 60.2 & 53.4 & 51.0 & 49.9 \\
\hline
\end{tabular}

sectores público y privado, política mundial, academia y sociedad civil.

\subsubsection{Retos y desafíos en el contexto actual mexicano.}

Es de suma importancia que en los países se implemente industria 4.0; ya que los avances en tecnología que trae consigo cada revolución es importante para ser competitivos. No es elección. Es indispensable. Las empresas que no implementen estas tecnologías lamentablemente morirán o se extinguirán en esta industria 4.0 ya que no solo los cambios se dan al interior de las empresas, sino que también en un ambiente sociocultural. (Tortorella, Cawley, \& Garza. 2019).

Es importante también que las instituciones de educación y el gobierno produzcan conocimiento de manera que les permita a las organizaciones tener personal que este altamente calificado en estas avanzadas tecnologías. (Peña \& Palacio. 2018).

México centra el uso de las tecnologías digitales 4.0 en herramientas administrativas y de comunicación y una parte menor en aplicaciones automatizadas y procesos de control para lo cual México es uno de los países de la OCDE con un porcentaje muy bajo del uso de servicios en la nube. Esta tecnología es considerada habilitadora como parte de la industria 4.0 la cual sirve de almacenamiento de recursos a los cuales se puede tener acceso

(Miles de millones de pesos de 2021)

Fuente: Centro de Estudios de Finanzas Públicas

Elaboración propia.

Es fundamental que México deba generar una inversión mayor en educación ya que de acuerdo a estadísticas emitidas por la Organización de las Naciones Unidas para la educación, la Ciencia y la Cultura (UNESCO), México dedico únicamente el $0.5 \%$ de su Producto Interno Bruto (PIB) a las áreas de investigación y desarrollo, en el año 2019.

Por otro lado, según el INEGI, el presupuesto en 2020 para Ciencia, Tecnología e Innovación (CTI), en México, es de 98 mil 317 millones de pesos, equivalente a tan solo el $0.38 \%$ del Producto Interno Bruto (PIB) de México.

En México, existe un organismo denominado Consejo Nacional de Ciencia y Tecnología (CONACYT), que, de acuerdo a la Ley General de Educación, Articulo 25, declara, que el Estado debe invertir como mínimo un $8 \%$ del PIB en material de Educación, del cual el 1\% debe dirigirse a la investigación científica y el desarrollo tecnológico realizado por instituciones de educación superior públicas. Es decir, es el organismo que regula, distribuye y administra los fondos para la producción del 
conocimiento científico.

A continuación, podemos ver la evolución del presupuesto destinado a la función Ciencia, Tecnología e Innovación que, en los últimos 10 años, ha sido ejercido el más bajo presupuesto registrado.

Como ha sido señalado por Cortés y Landeta, la búsqueda de generación de conocimiento y de mejora continua es constante, pero para llevarlo a las Instituciones requieren de apoyos y presupuestos. Ante tales situaciones, es de igual manera importante destacar los diversos cambios que han surgido en las políticas públicas nacionales impactan de manera directa en las instituciones educativas que reciben apoyos gubernamentales para el fortalecimiento de su planta docente e investigadores, sin embargo, la necesidad es imperante por ello la búsqueda de las diversas fuentes de financiamiento para consolidar proyectos nacientes y en desarrollo.

\section{MÉTODO}

El presente trabajo de investigación se elaboró una metodología de corte exploratorio y descriptiva, donde se analizaron temas que involucran el Modelo Triple Hélice y la Industria 4.0.

El concepto de investigación descriptiva ha sido definido por Carlos Sabino (1992) como un tipo de investigación que busca como objetivo principal el describir algunas características que sean esenciales para los conjuntos homogéneos de fenómenos, utilizando criterios sistemáticos que permiten establecer una estructura o comportamiento de fenómenos en estudio, proporcionando información sistémica y comparable con otras fuentes (Martínez, 2018).

Se llevó a cabo la revisión de literatura disponible en repositorios científicos, tales como, Redalyc, Scielo, Dialnet, Google Scholar. Y con ello obtener el acervo que nos permitió referenciar y fortalecer la creación de este artículo.

La selección y evaluación de la literatura se centró en criterios basados en el foco central de las investigaciones y validez de las fuentes de información.

Con el objeto de dar sustento a criterios que caracterizan al estudio aquí presentado se recurren como ya se mencionó anteriormente al tipo descriptivo, que refiere Sampieri y que busca especificar características, propiedades y perfiles de personas, grupos, comunidades, procesos, objetos o cualquiera de los fenómenos que se sometan a un análisis.

La realización de esta investigación permitió dar un enfoque a la información disponible y evidenciar la vinculación del Modelo Triple Hélice como una nueva forma de innovación que coadyuva y suma a las necesidades de las organizaciones de involucrarse en el desarrollo de temas alineados con la Industria 4.0, logrando así nuevas y mejores formas de gestión.

\section{CONCLUSIONES}

Los alcances del modelo de la Triple Hélice se centran en aportaciones teóricas y el marco de referencia de las economías basadas en conocimientos, que permiten ejercer nuevas fuentes y vías de desarrollo para la innovación.

Es evidente que el Modelo de la TH es perfectible de incorporar nuevas vertientes y procesos, pero la evidencia documental, reconoce la interacción y el impacto de los principales actores en los procesos de innovación.

El modelo de la TH genera una gran infraestructura de innovación, desarrollo y conocimiento por ello la incorporación de los elementos de la Industria 4.0 constituyen de gran impacto para nuevas formas de gestión y vinculación, que permitan a las organizaciones generar estrategias para lograr una ventaja competitiva y permanencia en los 
entornos globales.

De igual manera permite generar innovación en los procesos y sortear las situaciones económicas presentes de tal forma que se puedan generar mejores condiciones de desarrollo sustentable y sostenible con el entorno, en los diferentes ámbitos. Actualmente nuestro país atraviesa un déficit notable, en la aportación presupuestal para la Investigación y Desarrollo. La generación de políticas públicas de impacto, son necesarias, ya que es evidente la necesidad de investigar y desarrollar capacidades y conocimientos.

Es oportuno mencionar y dejar para la reflexión y próximas investigaciones el hecho de incluir un cuarto actor que puede conectarse al modelo tradicional de la TH y es específicamente la sociedad. Esto como es señalado por el mismo Leydesdorff, 2012, de que la ausencia de un traslape y la caracterización como positivo o negativo: "ofrece la posibilidad de que exista otro mecanismo sinérgico, a saber, el ajuste mutuamente selectivo de los ciclos en el curso del tiempo". La introducción de la "sociedad civil" en el modelo como la cuarta hélice, que "las hélices representan la especialización y la codificación en los sistemas funcionales que evolucionan a desde el seno de una sociedad civil. Sierra, J. (2018).

Cabe resaltar que, a pesar de las políticas públicas de cada uno de los países emergentes como México, muchas organizaciones de estos países para mejorar sus procesos han implementado tecnologías avanzadas y que pese a que no cuentan con los suficientes recursos para el desarrollo de las tecnologías; algunas han invertido en adquirir sistemas que cuentan con ciberseguridad para la protección de los datos.

Es también de suma importancia que los gobiernos deben trabajar de manera conjunta con las empresas y las instituciones de alta calidad con el único fin de mejorar planes de aprendizaje ya que como se comenta no se cuenta con las competencias necesarias para ocupar puestos calificados altamente en desarrollo de tecnologías que se ocupen en la mejora del aprendizaje. 


\section{REFERENCIAS}

Bueno, J. L. M. (2017). El Modelo triple hélice de innovación: importancia teórica y evidencias de su aplicación en el desarrollo de la innovación. Catequil Tekné, 1(01), 41-53.

Casalet, M. y Stezano, F. (2020). Riesgos y oportunidades para el avance de la digitalización en México. Economía de la innovación y las nuevas tecnologías, 29 (7), 689-704.

Chang Castillo, H. G. (2010). El modelo de la triple hélice como un medio para la vinculación entre la Universidad y la Empresa. Revista Nacional De Administración, 1(1), 85-94. https://doi.org/10.22458/rna.v1i1.286

COMISION EUROPEA, 2013, “Comprender las políticas de la unión europea. Una nueva revolución industrial”, Comisión Europea-Empresa, 1-16.

Cortés, C. B. Y., Landeta, J. M. I., \& Chacón, J. G. B. (2017). El entorno de la industria 4.0: implicaciones y perspectivas futuras. Conciencia tecnológica, (54), 33-45.

De la Fe, T. G. (2009). El modelo de triple hélice de relaciones universidad, industria y gobierno: un análisis crítico. Arbor, 185(738), 739-755.

Del Val Román, J. L. (2016, March). Industria 4.0: la Transformación digital de la Industria. In Valencia: Conferencia de Directores y Decanos de Ingeniería Informática, Informes CODDII.

Etzkowitz, H. (2000a). The triple helix of university-industry-government: implications for policy and evaluation. Swedish Institute for Studies in Education and Research, Stockholm (Sweden).

Etzkowitz, H. (2002b). Networks of innovation: science, technology and development in the triple helix era. International Journal of Technology Management \& Sustainable Development, 1(1), 7-20.

Etzkowitz, H. y Leydesdorff, L. (1995). The triple helix university-industry-government relations: A laboratory for knowledge based economic development. Easst Review, 14(1), 14-19.

Etzkowitz, H. y Leydesdorff, L. (1998). The triple helix as a model for innovation studies. Science and public policy, 25(3), 195-203.

Etzkowitz, H. y Leydesdorff, L. (2000). The dynamics of innovation: from National Systems and "Mode 2" to a Triple Helix of university-industry-government relations. Research policy, 29(2), 109-123.

Evans, P y Annunziata, M. (2012): "Industrial Internet: Pushing the Boundaries of Minds and Machines". Imagination at work, 1-37.

Fernando Franco, D. (2015). Utilización del Modelo de Triple Hélice para el desarrollo de nuevos sectores productivos en el contexto de la Industria 4.0.

Hernández, L. C., Verástegui, J. L., \& Melo, N. A. P. (2014). La gestión de la triple hélice: fortaleciendo las relaciones entre la universidad, empresa, gobierno. Multiciencias, 14(4), 438446.

Hernández Sampieri, R. (2016). Metodología de la Investigación. México: McGraw Hill.

Kagermann, H.; Wahlster, W. y Helbig, J. (2013): "Recommendations for implementing the strategic initiative INDUSTRIE 4.0". Acatech, 1-82.

Luengo, M. J., \& Obeso, M. (2013). El efecto de la triple hélice en los resultados de innovación. Revista de Administração de Empresas, 53(4), 388-399.

Martínez, C. (2018). Investigación descriptiva: definición, tipos y características. Obtenido de https://www.lifeder.com/investiga-cion-descriptiva

Peña, O. L., \& Palacio, G. J. (2018). Impacto de las nuevas tecnologías de industry 4.0 en Colombia. Loginn Vol. 2 Numero 2.

Rubiano Valbuena, M. A. (2020). Industria 4.0.

Sierra, J. (2018). La cuarta hélice y la financiación de la innovación. Journal of Economics, Finance and Administrative Science

SPRI (2015a): "Industrie 4.0 -¿Utopía digital o business case?- Basque Industry 4.0”[Video] Edita YouTube. ( https://www.youtube.com/watch?v=9dtxBsSeinw )

Tortorella, G. L., Cawley, A., \& Garza, J. A. (2019). Organizational learning paths based upon industry 4.0 adoption An empirical study with brazilian manufacturers. International Journal 
of Production Economics. 